\title{
The Use of a Weight-Bearing Biofeedback System as an Adjunct in Rehabilitation Following a Simultaneous Bilateral Anterior Cruciate Ligament Reconstruction: A Case Study Report
}

Yonatan Kaplan* and Noa Spieler

Lerner Sports Center, Hebrew University of Jerusalem, 1 Churchill St., Mount Scopus, Jerusalem, IL, Israel

\begin{abstract}
Background: Despite the plethora of published articles relating to anterior cruciate ligament injuries, little evidence exists regarding the subject of simultaneous bilateral anterior cruciate ligament reconstruction (SBACLR) and its rehabilitation challenges.

Case Report: This case study presents a 17-year-old female athlete who underwent a rare surgical procedure: a SBACLR and suturing of the medial collateral ligament of one knee. A relatively new innovative computerized air-insole auditory biofeedback system (Smartstep ${ }^{\mathrm{TM}}$ ) was used as an adjunct in the evaluation and promotion of weight-bearing in the early stages of rehabilitation.
\end{abstract}

Results: Following intensive rehabilitation over a seven-week period, the athlete progressed in all functional activities of daily living, with a gradual bilateral improvement in weight-bearing abilities. After completion of her rehabilitation program, she returned symptom-free to all previous functional and sporting activities.

Conclusions: This case study highlights the fact that although a SBACLR is still a relatively uncommon knee surgical procedure, the possibility exists for an athlete to return to full functional activities of daily living. The Smartstep ${ }^{\mathrm{TM}}$ system proved to be a useful objective and accurate adjunct during the rehabilitation process.

Keywords: Knee; Anterior cruciate ligament reconstruction; Weightbearing; Biofeedback

Abbreviations: ACL: Anterior Cruciate Ligament; ACLR: Anterior Cruciate Ligament Reconstruction; SBACLR: Simultaneous Bilateral Anterior Cruciate Ligament Reconstruction; MCL: Medial Collateral Ligament; LQF: Lysholm Questionnaire Form; TALS: Tegner Activity Level Scale; IKDC: International Knee Documentation Committee

\section{Introduction}

One of the more common injuries in sports that involve sudden directional changes is the rupture of the anterior cruciate ligament (ACL) within the knee. Patients who have bilateral ACL-deficient knees are occasionally seen in a sports medicine practice. Despite reports of simultaneous bilateral ACL ruptures in the literature, the vast majority of patients presenting with bilateral ACL deficient knees sustain no simultaneous bilateral ACL ruptures [1]. ACL injury occurs four to six times more frequently in women than in men who had participated in the same sporting activity, a phenomenon that is well-supported by the literature $[2,3]$. The reasons postulated for this difference include neuromuscular, hormonal and anatomical factors $[4,5]$.

Without anterior cruciate ligament reconstruction (ACLR), there tends to be a significant increase in the risk for further meniscal damage [6] and a decrease in the proprioceptive function of the knee joint [4]. The rehabilitation process following ACLR ranges between four to six months [7]. In the case of a bilateral ACL tear, where the reconstruction is carried out on each knee separately, the athlete will be unable to return to play for approximately a year. The incidence of bilateralism is reported to be between two and four percent of ACLinjured knees $[1,8]$. ACLR results in changes in lower extremity joint kinetics, including in the normal gait pattern [9]. These changes include an abnormal lower leg movement pattern, a stiffening strategy of the knee and a reduced knee range of movement [9-12].

There is no consensus regarding the optimal rehabilitation protocol following ACLR and many varying protocol paradigms have been proposed $[13,14]$. The authors conducted a literature search of all articles written in English. Six articles were found that discussed the subject of simultaneous bilateral anterior cruciate ligament reconstruction (SBACLR) [1,15-19]. One study, [17] reported on a case series of eight patients who underwent simultaneous bilateral ACL reconstruction. The authors concluded that when there is a need to perform this type of operation, it is a safe and clinically effective option that is time and economically sound. The most recent prospective comparative study compared the mid-term outcome of 7 patients (14 knees) who had one-stage bilateral ACL reconstruction with that of a matched group of patients who had unilateral reconstruction (21 patients). Their mid-term clinical results suggested that one-stage bilateral ACL reconstruction using either hamstring or patella tendon autograft is clinically effective, reproducible, cost effective and does not compromise functional results. Both these studies had very small patient cohorts and their follow up period was limited, so no long term conclusions may be drawn. Little detail was provided related to the rehabilitation protocol used in the studies.

Asymmetric gait patterns persist up to one year post-ACLR and are more pronounced during stair ascent and descent than in level walking. These results indicate that clinicians should include specific interventions aimed at improving knee function during stair use in order to restore normal function after ACLR [20]. It may take up

*Corresponding author: Yonatan Kaplan, Jerusalem Sports Medicine Institute Lerner Sports Center, Hebrew University of Jerusalem, 1 Churchill St., Mount Scopus, Jerusalem, Israel, Tel: 972-2-502-3941; Fax: +972-2-581-2102; E-mail: sportmed@zahav.net.il; www.jsportmed.com

Received May 26, 2014; Accepted September 01, 2014; Published September 13, 2014

Citation: Kaplan Y, Spieler N (2014) The Use of a Weight-Bearing Biofeedback System as an Adjunct in Rehabilitation Following a Simultaneous Bilateral Anterior Cruciate Ligament Reconstruction: A Case Study Report. J Sports Med Doping Stud 4: 145. doi:10.4172/2161-0673.1000145

Copyright: $\odot 2014$ Kaplan Y, et al. This is an open-access article distributed under the terms of the Creative Commons Attribution License, which permits unrestricted use, distribution, and reproduction in any medium, provided the original author and source are credited. 
Citation: Kaplan Y, Spieler N (2014) The Use of a Weight-Bearing Biofeedback System as an Adjunct in Rehabilitation Following a Simultaneous Bilateral Anterior Cruciate Ligament Reconstruction: A Case Study Report. J Sports Med Doping Stud 4: 145. doi:10.4172/21610673.1000145

to a year to achieve normalized gait biomechanics in these patients $[1,21]$. Early normal gait restoration has shown not only to be safe, but essential to rapidly regain normal muscle function and to significantly lower post-surgical complications, such as knee stiffness [22,23]. As a result, it is accepted that early full weight-bearing should be a primary rehabilitative goal from almost immediately post-ACLR [22,24]. In order to improve weight-bearing deficits following ACLR, it is initially required to ascertain the magnitude of these deficits. This becomes especially challenging when bilaterality exists and there is no healthy limb for weight-bearing comparison. This was the primary purpose of utilizing the Smartstep ${ }^{\text {tw }}$ system $^{\star}$. The SmartStep ${ }^{\text {Tw }}$ pneumatic insole system measures key gait parameters during ambulation. The data are received and then analyzed by the miniature portable microprocessor, which is worn around the ankle. This is subsequently transmitted to a computer system running the SmartStep ${ }^{\text {Tw }}$ software, which also maintains patient's medical records. The software additionally functions as an assessment of gait analysis, which includes weight-bearing distribution, stance/swing phase and cadence values. The device further contains a biofeedback training application for rehabilitation. The main advantage of this system over other laboratory measuring devices lies in its ability to measure weight-bearing parameters in various functional settings, for example, walking, running and stair negotiation. These above mentioned devices are expensive, with measurement apparatuses that are not commonly portable. Additionally, they are not readily available in clinical and rehabilitation settings and cannot be adapted for usage in popular sporting activities

The aim of this case report was to describe for the first time, the use of a relatively new innovative computerized air-insole auditory biofeedback system as an adjunct in the evaluation and restoration of functional activity (symmetric gait patterns, weight-bearing and biomechanics) in the early stages of rehabilitation following a SBACLR. Clinical recommendations were provided.

${ }^{*}\left(\right.$ Smartstep ${ }^{\text {tx }}$ System, Andante Medical Devices Ltd, Omer Industrial Park, bld. 8b, Omer 84965, Israel. info@andante.co.il).

\section{Case Report}

\section{Patient history}

L.L., a seventeen yr. old girl from Cambodia, was referred to our clinic, 3-weeks post-ACLR. She was a very active athlete and had competed in different sports from a young age, including basketball, handball, soccer, and hockey. A year prior to the surgery, she had fallen during a basketball game and had twisted her right knee. She was diagnosed by a local orthopedic surgeon as having sustained a complete rupture of her right ACL. After a month's course of physical therapy treatment, she returned to full sporting activity. Six months later, while playing hockey, she was pushed, and subsequently twisted her left knee. Following a clinical examination, she was diagnosed with a complete rupture of her ACL, with a suspicion of a complete rupture of her MCL. After the injury, she once again underwent physical therapy rehabilitation; however she was unable to return to sporting activities.

In order to evaluate her functional ability, the Lysholm questionnaire form (LQF) [25], Tegner Activity Level Scale (TALS) [26] and the International Knee Documentation Committee (IKDC) [27] scores were used at different stages of her rehabilitation process. Her pre-surgery results indicated a gross deficit in functional activity (Table 1). It was agreed to perform a SBACLR and an MCL repair in Sweden. The operation was performed using the bone-patella-bone method. The referral report received by the authors indicated that her rehabilitation program started four days post-surgery for a period of three weeks. She was instructed to ambulate as tolerated with the use of Canadian elbow crutches and to wear bilateral knee braces. She performed closedkinetic chain hamstring and quadriceps strengthening exercises over this period.

\section{Rehabilitation program}

When the patient arrived at the Lerner Sports Center, Hebrew University, three weeks post-surgery, she was still ambulating with crutches, as well as wearing bilateral knee braces (Figure 1).

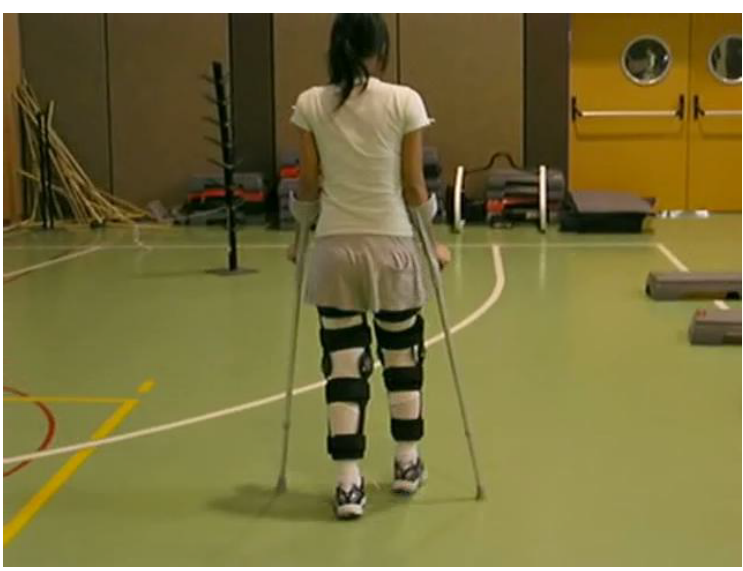

Figure 1: Athlete Walking on Admission.

\begin{tabular}{|c|c|c|c|c|c|c|c|c|c|c|c|c|}
\hline \multicolumn{9}{|c|}{ Knee range of movement } & \multicolumn{4}{|c|}{$\begin{array}{l}\text { Quadriceps strength (Kg/force)Hand-held Dynamometer- } \\
\text { (Jamar®, Sammons Preston, Inc) }\end{array}$} \\
\hline \multicolumn{5}{|c|}{ End of week 3} & \multicolumn{4}{|c|}{ End of week 7} & \multicolumn{2}{|c|}{ End of week 3} & \multicolumn{2}{|c|}{ End of week 7} \\
\hline & \multicolumn{2}{|c|}{ Right } & \multicolumn{2}{|c|}{ left } & \multicolumn{2}{|c|}{ right } & \multicolumn{2}{|c|}{ left } & right & left & right & left \\
\hline Extension & \multicolumn{2}{|c|}{$0^{\circ}$} & \multicolumn{2}{|c|}{$0^{\circ}$} & \multicolumn{2}{|c|}{$0^{\circ}$} & \multicolumn{2}{|c|}{$0^{\circ}$} & 11 & 12 & 28 & 25 \\
\hline \multirow[t]{3}{*}{ Flexion } & \multicolumn{2}{|c|}{$120^{\circ}$} & \multicolumn{2}{|c|}{$80^{\circ}$} & \multicolumn{2}{|c|}{$140^{\circ}$} & \multicolumn{2}{|c|}{$140^{\circ}$} & & & & \\
\hline & & & & & & & & & Average & $t \%$ bc & eight-be & alues \\
\hline & \multicolumn{2}{|c|}{ Pre-surgery } & \multicolumn{2}{|c|}{ End of week 3} & \multicolumn{2}{|c|}{ End of week 7} & \multicolumn{2}{|c|}{ At one year } & \multicolumn{2}{|c|}{ End of week 3} & \multicolumn{2}{|c|}{ End of week 7} \\
\hline \multirow[t]{2}{*}{ Lysholm score } & right & left & right & left & right & left & right & left & \multirow{2}{*}{ right } & \multirow{2}{*}{ left } & \multirow{2}{*}{ right } & \multirow{2}{*}{ left } \\
\hline & 68 & 50 & 62 & 58 & 88 & 76 & 100 & 100 & & & & \\
\hline IKDC score & 50 & 35 & 65 & 50 & 90 & 70 & 101 & 101 & $62 \%$ & $68 \%$ & $96 \%$ & $108 \%$ \\
\hline TALS & \multicolumn{2}{|c|}{2} & \multicolumn{2}{|c|}{3} & \multicolumn{2}{|c|}{5} & \multicolumn{2}{|c|}{10} & & & & \\
\hline
\end{tabular}

Table 1: Measurements During Rehabilitation. 
Citation: Kaplan Y, Spieler N (2014) The Use of a Weight-Bearing Biofeedback System as an Adjunct in Rehabilitation Following a Simultaneous Bilateral Anterior Cruciate Ligament Reconstruction: A Case Study Report. J Sports Med Doping Stud 4: 145. doi:10.4172/21610673.1000145

On initial examination, it was difficult to ascertain definite, objective lower-limb atrophy or swelling because of the inability to establish a comparison with the contralateral uninjured side. A summary of her muscle strength tests, range of motion, LQF and TALS and IKDC scores at initial examination are summarized in Table 1.

Her physiotherapy program consisted of three treatment sessions per week, each lasting for 90 minutes, over a period of four weeks. Her initial rehabilitation protocol included individual sessions with either one of the authors and was based upon the accelerated protocol, which has been previously published in the literature [7]. It consisted of lower-limb open and closed chain strengthening exercises, electrical muscle stimulation and neuromuscular sensory training. The program then progressed to simple polymeric and sportmetric-type exercises and perturbation training on uneven surfaces. As an adjunct to this program, starting from the initial treatment, she had six sessions with the SmartStep ${ }^{\text {Tx }}$ weight-bearing system (Figure 2) which was used both as a weight-bearing monitoring system as well as a biofeedback training mechanism. This was done in order to improve and encourage her weight-bearing until she had achieved equal full weight-bearing bilaterally. She provided informed consent to the use of the Smartstep apparatus during her rehabilitation program as well as to her participation in this case study.

In the first evaluation with the Smartstep ${ }^{\mathrm{sm}}$ system (during the fourth week of her rehabilitation), she was instructed to walk at a normal pace over a hard surface for a 10 meter distance, while the Smartstep ${ }^{\text {tw }}$ system measured her weight-bearing status. The results of this evaluation

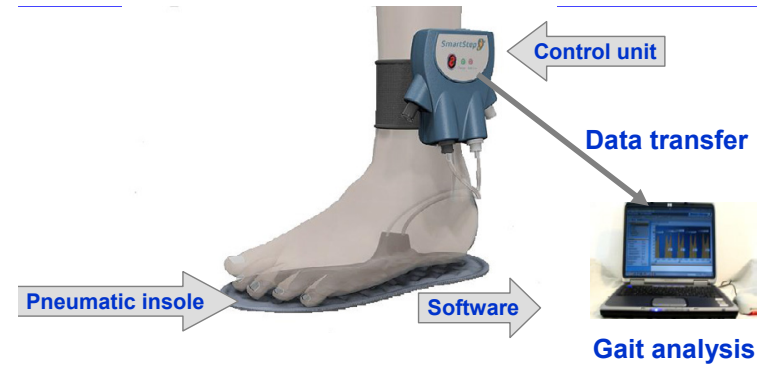

Figure 2: The Computerized Air-insole Auditory Biofeedback System $\left(\right.$ Smartstep $\left.{ }^{\mathrm{TM} *}\right)$

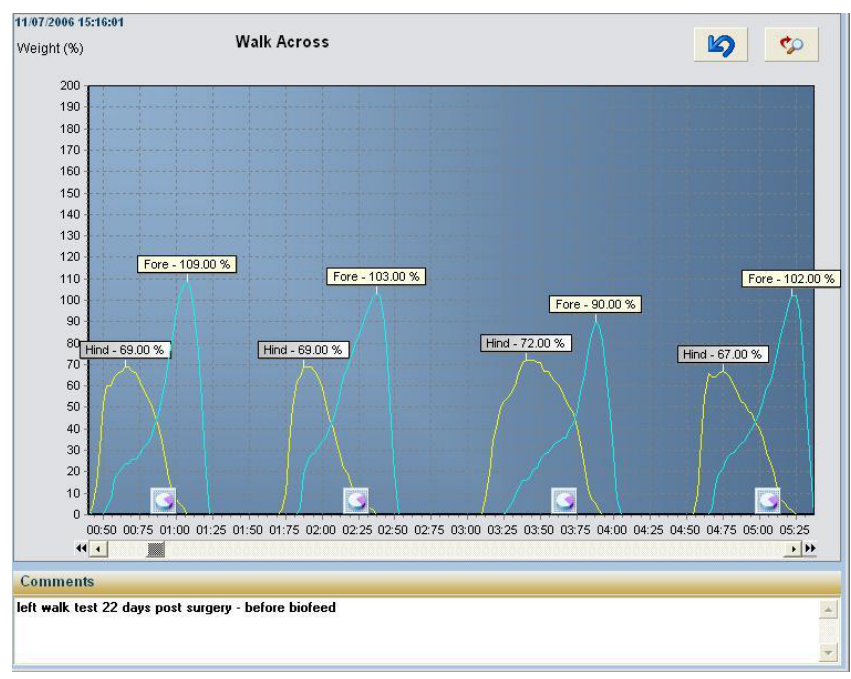

Figure 3: Analysis of the first test result.

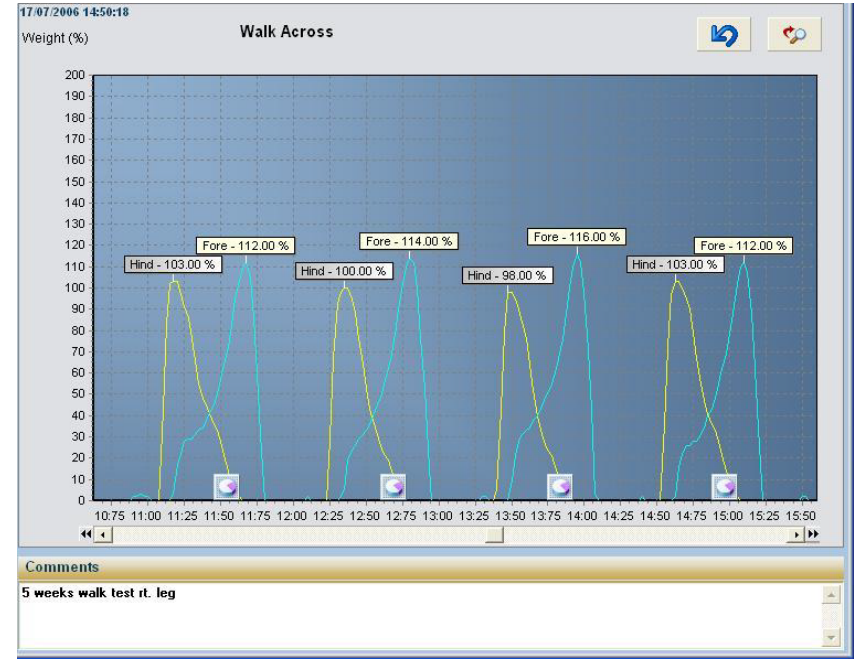

Figure 4: Analysis of the post-biofeedback training result

(Table 1) showed that her bilateral hind-foot weight-bearing values were significantly reduced (Figure 3 ). The normal average percentage hind-foot body-weight values in walking have been reported to be $82.33 \%$ [28].

\section{Results}

After two weeks of rehabilitation in the Lerner Center, L.L. reached full, equal weight-bearing bilaterally (Figure 4). At seven weeks postsurgery, she displayed a marked improvement in her LQS, TALS and IDKC scores. After four weeks of rehabilitation in the Center, she returned to Sweden, where she completed her rehabilitation. She returned for a visit one year later and reported a full and symptom-free return to all previous functional and sporting activities (Table 1).

\section{Discussion}

This is the first case study in the literature describing the use of the Smartstep $^{\text {Tu }}$ system as an adjunct therapy post - ACL reconstruction. The Smartstep ${ }^{\text {TI }}$ system indeed provided initial evidence that it was an accurate and objective system, both in terms of its measurement parameters, as well as its biofeedback capability in rehabilitation following SBACLR. It further enabled an accurate comparison to normal weight-bearing values and gait characteristics, without relying on the unaffected limb alone for comparison.

The case study adds somewhat to the limited evidence currently available in the literature, that SBACLR is a safe and clinically effective treatment modality for patients presenting with symptomatic bilateral ACL deficiency. There was a marked functional improvement in the patient's progress over a relatively short time period of seven weeks. The authors were able to take advantage of the profusion of evidence relating to the efficacy of different treatment protocols in the treatment of a unilateral ACLR, and utilized previously described treatment regimes. This rehabilitation strategy when applied to bilateral reconstructions relies on the concept that while one knee is undergoing rehabilitation, the other knee is also being rehabilitated.

Shelbourne [7] has emphasized the importance of regaining symmetrical knee strength and function in achieving early return to function [24]. The rehabilitation program undertaken by the patient in this case study embraced this philosophy. She was able to return to full, unrestricted activity involving cutting and pivoting activities, 
Citation: Kaplan Y, Spieler N (2014) The Use of a Weight-Bearing Biofeedback System as an Adjunct in Rehabilitation Following a Simultaneous Bilateral Anterior Cruciate Ligament Reconstruction: A Case Study Report. J Sports Med Doping Stud 4: 145. doi:10.4172/21610673.1000145

where normal and equal strength are required bilaterally. This is in concurrence with the results of studies indicating that there was no observable difference between the recovery time of patients undergoing unilateral surgery and those undergoing bilateral ACL reconstruction [15].

Many surgeons are unwilling to consider SBACLR, as they wish their patients to have an unoperated leg following surgery. This is in order to prevention potential complications during the procedure or during the rehabilitation process thereafter.

There are several challenges that the rehabilitation team faces following a SBACLR. The primary difficulty being the objective evaluation and measurement between the two limbs throughout the rehabilitation process. It remains difficult to obtain an objective comparison pertaining to the swelling, strength and neuromuscular differences between the two legs, as there is no "healthy" contralateral side for comparison. Additionally, there is no paradigm upon which to base the rehabilitation, as it has not been previously reported in the literature.

This is a single case study only, and its conclusions should provide the basis of a future long term prospective study including a much larger cohort of patients who have undergone SBACLR. A randomised control trial may be undertaken comparing the results gained by using the SmartStep system early in rehabilitation vs. a control group. The clinical relevance of a potentially reduced period of rehabilitation by doing the one-stage SBACLR, as well as reduced anesthetic and operating time when compared to staged or non-simultaneous bilateral ACL reconstruction, are important considerations that require further study. Is must be noted however, that Jari \& Shelbourne15 have emphasized that severe bilateral quadriceps weakness may be a relative contraindication to bilateral ACL reconstruction.

\section{Conclusions}

The Smartstep ${ }^{\mathrm{Tm}}$ system proved to be a useful objective and accurate adjunct during the rehabilitation process. This study demonstrates that following this relatively uncommon operative procedure, an SBACLR and an MCL repair, it is possible to return an athlete to full, active, functional activities of daily living, together with a high-level of sporting activity. What is evident however is the need for more studies to be conducted in order to further optimize rehabilitation protocols after ACLR in general and in particular, following the SBACLR procedure.

\section{References}

1. Souryal TO, Moore HA, Evans JP (1988) Bilaterality in anterior cruciate ligament injuries: associated intercondylar notch stenosis. Am J Sports Med 16: $449-454$

2. Hewett TE (2000) Neuromuscular and hormonal factors associated with knee injuries in female athletes. Strategies for intervention. Sports Med 29: 313-327.

3. Myer GD, Ford KR, Paterno MV, Nick TG, Hewett TE (2008) The effects of generalized joint laxity on risk of anterior cruciate ligament injury in young female athletes. Am J Sports Med 36: 1073-1080.

4. Hewett TE, Myer GD, Ford KR (2004) Decrease in neuromuscular control about the knee with maturation in female athletes. J Bone Joint Surg Am 86-86A: 1601-1608.

5. Hewett TE, Myer GD, Ford KR (2006) Anterior cruciate ligament injuries in female athletes: Part 1, mechanisms and risk factors. Am J Sports Med 34: 299-311.

6. Fithian DC, Paxton EW, Stone ML, Luetzow WF, Csintalan RP, et al. (2005) Prospective trial of a treatment algorithm for the management of the anterior cruciate ligament-injured knee. Am J Sports Med 33: 335-346.

7. Shelbourne KD, Nitz $P(1990)$ Accelerated rehabilitation after anterior cruciate ligament reconstruction. Am J Sports Med 18: 292-299.
8. Anderson AF, Lipscomb AB, Liudah KJ, Addlestone RB (1987) Analysis of the intercondylar notch by computed tomography. Am J Sports Med 15: 547-552

9. Kurz MJ, Stergiou N, Buzzi UH, Georgoulis AD (2005) The effect of anterior cruciate ligament reconstruction on lower extremity relative phase dynamics during walking and running. Knee Surg Sports Traumatol Arthrosc 3: 107-115.

10. Fitzgerald GK, Axe MJ, Snyder-Mackler L (2000) A decision-making scheme for returning patients to high-level activity with nonoperative treatment after anterior cruciate ligament rupture. Knee Surg Sports Traumatol Arthrosc 8: 7682.

11. Rudolph KS, Axe MJ, Buchanan TS, Scholz JP, Snyder-Mackler L (2001) Dynamic stability in the anterior cruciate ligament deficient knee. Knee Surg Sports Traumatol Arthrosc 9: 62-71.

12. Knoll Z, Kocsis L, Kiss RM (2004) Gait patterns before and after anterior cruciate ligament reconstruction. Knee Surg Sports Traumatol Arthrosc 12: 7-14.

13. Decker MJ, Torry MR, Noonan TJ, Sterett WI, Steadman JR (2004) Gait retraining after anterior cruciate ligament reconstruction. Arch Phys Med Rehabil 85: 848-856.

14. Myer GD, Paterno MV, Ford KR, Quatman CE, Hewett TE (2006) Rehabilitation after anterior cruciate ligament reconstruction: criteria-based progression through the return-to-sport phase. J Orthop Sports Phys Ther 36: 385-402.

15. Jari S, Shelbourne KD (2002) Simultaneous bilateral anterior cruciate ligament reconstruction. Am J Sports Med 30: 891-895.

16. Larson CM, Fischer DA, Smith JP, Boyd JL (2004) Bilateral Anterior Cruciate Ligament reconstruction as a single procedure: evaluation of cost and early functional results. Am J Sports Med 32: 197-200.

17. Saithna A, Arbuthnot J, Carey-Smith R, Spalding T (2010) Simultaneous bilateral anterior cruciate ligament reconstruction: a safe option. Knee Surg Sports Traumatol Arthrosc 18: 1071-1074.

18. Sajovic M, Demsar S (2007) One-stage bilateral anterior cruciate ligament reconstruction with use of hamstring tendon autografts: a case report. Knee Surg Sports Traumatol Arthrosc 15: 1114-1115.

19. Sajovic M, Demsar S, Sajovic R (2013) One-stage bilateral anterior cruciate ligament reconstruction. Knee Surg Sports Traumatol Arthrosc 21: 1998-2003.

20. Hooper DM, Morrissey MC, Drechsler W, Morrissey D, King J (2001) Open and closed kinetic chain exercises in the early period after anterior cruciate ligament reconstruction. Improvements in level walking, stair ascent, and stair descent. Am J Sports Med 29: 167-174.

21. Timoney JM, Inman WS, Quesada PM, Sharkey PF, Barrack RL, et al. (1993) Return of normal gait patterns after anterior cruciate ligament reconstruction. Am J Sports Med 21: 887-889.

22. Shelbourne KD, Nitz $P$ (1990) Accelerated rehabilitation after anterior cruciate ligament reconstruction. Am J Sports Med 18: 292-299.

23. Shelbourne KD, Wilckens JH (1990) Current concepts in anterior cruciate ligament rehabilitation. Orthop Rev 19: 957-964

24. McCarroll JR, Shelbourne KD, Patel DV (1995) Anterior cruciate ligament injuries in young athletes. Recommendations for treatment and rehabilitation. Sports Med 20: 117-127.

25. Marx RG, Jones EC, Allen AA, Altchek DW, O'Brien SJ, et al. (2001) Reliability, validity, and responsiveness of four knee outcome scales for athletic patients. $J$ Bone Joint Surg Am 83-83A: 1459-69.

26. Tegner Y, Lysholm J (1985) Rating systems in the evaluation of knee ligament injuries. Clin Orthop Relat Res : 43-49.

27. Irrgang JJ, Ho H, Harner CD, Fu FH (1998) Use of the International Knee Documentation Committee guidelines to assess outcome following anterior cruciate ligament reconstruction. Knee Surg Sports Traumatol Arthrosc 6: 107114.

28. Kaplan Y, Spieler N, Barak Y (2008) Normal percentage body-weight/ weightbearing values and gait characteristics in activities of daily living: An original research study. Gait Posture 28: 50 . 